\title{
REVISION OF THE FORMULÆ OF WEINSTEIN AND STEFAN FOR THE MUTUAL INDUCTANCE OF COAXIAL COILS.
}

\author{
By Edward B. Rosa.
}

The only formulæ for the mutual inductance of two coaxial coils of rectangular cross section that have been proposed which take account of differential coefficients higher than the second are those of Weinstein ${ }^{1}$ and Stefan. ${ }^{2}$ The formulæ of Ruwland, ${ }^{3}$ Rayleigh, ${ }^{4}$ and Lyle ${ }^{5}$ take account of second differentials only, and while sufficiently accurate for coils of relatively small section and considerable distance apart are not so accurate for coils of larger cross section, or for coils of small section if they are relatively near each other. The formulæ of Weinstein and Stefan seem to have been very little employed, and so far as I know have not been critically examined or compared with one another. The former of these two formulæ is given on page 340 , of this paper, equation (I4)-the latter is (2I) of page 342 .

Weinstein derived his formula by starting with Maxwell's expression in elliptic integrals for the mutual inductance of two coaxial circles (equation I 3 below) and differentiating it, as Rowland did, carrying the operation, however, to the fourth order of differentials. Stefan, on the other hand, took Maxwell's second expression (equation 25 below), where the mutual inductance of two coaxial circles is expressed in a converging series, and differentiated it, or certain terms of it, to the sixth order. Omitting the terms in the latter depending on differentials higher than the fourth order, these two

${ }^{1}$ Wied. Annalen, 21, p. 329 ; I884.

${ }^{2}$ Wied. Annalen, 22, p. II5; I884.

${ }^{3}$ American Journal of Science, 15 ; 1878.

${ }^{4}$ Maxwell, Vol. II, Chapter XIV, Appendix II.

${ }^{5}$ Phil. Mag., 3, p. $310 ; 1902$. 
formulæ should give the same results. They do not, however, agree as closely as they ought, and examination shows that Weinstein's is very nearly exact when the coils are at considerable distance from one another, but less accurate when they are near. Stefan's, on the other hand, is very accurate when the coils are near but less accurate when they are far apart, the relative error increasing with the distance. The error in either case is small, and yet too large for precision work, and larger than can be explained by the terms of higher degree omitted in deriving the formulæ. In going over Weinstein's work, and in deriving Stefan's formula anew (Stefan gives only his result, without any indication of the process by which it was derived)

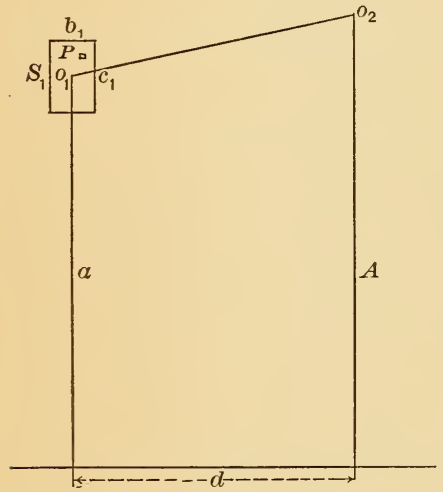

Fig. 1

I have found errors in both formulæ. These being corrected they agree as well as could be expected over a wide range of distances and for coils of considerable cross section. Stefan's formula can be put into a form that is easy for calculation, being much more conrenient than the other less accurate formulæ. Stefan's and Weinstein's formulæ are, however, limited by the condition that the two coils are of equal radii, whereas Rayleigh's and Lyle's apply also to coils of unequal radii.

As formulæ for the accurate calculation of the mutual inductance of parallel coils are of fundamental importance, and as these forinulæ of Weinstein and Stefan as revised and corrected are the most accurate formulæ in existence (for coils of equal radii), I shall give somewhat fully the derivation of Stefan's and the revision of Weinstein's, and shall put them into different forms, which are more convenient for calculation than the forms in which they were originally given.

\section{MUTUAL INDUCTANCE OF A CIRCLE AND A COIL.}

Let $\mathrm{O}_{2}$ be a circle of radius $\mathrm{A}$ and $\mathrm{S}_{1}$ be a coil of mean radius $\alpha$, axial breadth $b_{1}$, and radial depth $c_{1}$, the distance between the mean plane of the coil and the plane of the circle being $d$. We wish to find first the mutual inductance of the circle $\mathrm{O}_{2}$ and the coil $\mathrm{S}_{1}$. 
One way to proceed would be to take the expression for the mutual inductance of the circle $\mathrm{O}_{2}$ and a second parallel circle $\mathrm{P}$ of cross sectional area $d x d y$ within the section of the coil and integrate this over the area of the section $\mathrm{S}_{1}$. This is a difficult and complicated operation, and has never been done. Maxwell, ${ }^{6}$ however, shows how to get an approximate result more easily by means of Taylor's theorem, and this result can be made very accurate by taking a sufficient number of terms.

If $f(x, y)=u$, Taylor's theorem gives

$$
\begin{aligned}
f(x+h, y+k)= & u+\left(h \frac{d u}{d x}+k \frac{d u}{d y}\right)+\frac{\mathrm{I}}{\mathrm{I} \cdot 2}\left(h^{2} \frac{d^{2} u}{d x^{2}}+2 h k \frac{d^{2} u}{d x d y}+k^{2} \frac{d^{2} u}{d y^{2}}\right) \\
& +\frac{\mathrm{I}}{\mathrm{I} \cdot 2 \cdot 3}\left(h^{3} \frac{d^{3} u}{d x^{3}}+3 h^{2} k \frac{d^{3} u}{d x^{2} d y}+3 h k^{2} \frac{d^{3} u}{d x d y^{2}}+k^{3} \frac{d^{3} u}{d y^{3}}\right) \\
& +\ldots \ldots \ldots+\frac{\mathrm{I}}{n !}\left(h \frac{d}{d x}+k \frac{d}{d y}\right) u
\end{aligned}
$$

Applying this to the case of the circle and the coil, $h$ and $k$ would be the coordinates of the point $\mathrm{P}$ with $\mathrm{O}_{1}$ as origin, $u$ would be the mutual inductance of the circle $\mathrm{O}_{2}$ and the circle $\mathrm{O}_{1}$, and $f(x+h, y+k)$ would be the mutual inductance of the circle $\mathrm{O}_{2}$ and the circle $\mathrm{P}$. To get the mutual inductance of the circle $\mathrm{O}_{2}$ and the coil $\mathrm{S}_{1}$ it will be necessary to integrate this expression over the area of $S_{1}$ and divide by the area $b_{1} c_{1}$.

Using Maxwell's notation, the mean value $\bar{P}$ of any function $P$ of $x$ and $y$ is

$$
\bar{P}=\frac{\mathrm{I}}{x y} \int_{-\frac{x}{2}}^{+\frac{x}{2}} \int_{-\frac{y}{2}}^{+\frac{y}{2}} P d x d y
$$

This mean value is, of course, the mean for the region within the limits of integration, and if this operation be applied to the expression for mutual inductance of the two circles $\mathrm{O}_{2}$ and $\mathrm{P}$ we can obtain an expression for the mutual inductance of $\mathrm{O}_{2}$ and the coil $\mathrm{S}_{1}$ of section $b_{1} c_{1}$. Writing $M_{0}$ for the mutual inductance of $\mathrm{O}_{1}$ and $\mathrm{O}_{2}$, 
$M^{\prime}$ for the mutual inductance of $\mathrm{P}$ and $\mathrm{O}_{2}$, and $x$ and $y$ in place of $h$ and $k$, we have in place of $(\mathrm{I})$ :

$$
\begin{aligned}
& M^{\prime}=M_{0}+\left(x \frac{d M_{0}}{d x}+y \frac{d M_{0}}{d y}\right)+\frac{\mathrm{I}}{\mathrm{I} \cdot 2}\left(x^{2} \frac{d^{2} M_{0}}{d x^{2}}+2 x y \frac{d^{2} M_{0}}{d x d y}+y^{2} \frac{d^{2} M_{0}}{d y^{2}}\right) \\
& +\frac{\mathrm{I}}{3 !}\left(x^{3} \frac{d^{3} M_{0}}{d x^{3}}+3 x^{2} y \frac{d^{3} M_{0}}{d x^{2} d y}+3 x y^{2} \frac{d^{3} M_{0}}{d x d y^{2}}+y^{3} \frac{d^{3} M_{0}}{d y^{3}}\right) \\
& +\frac{1}{4 !}\left(x^{4} \frac{d^{4} M_{0}}{d x^{4}}+4 x^{3} y \frac{d^{4} M_{0}}{d x^{3} d y}+6 x^{2} y^{2} \frac{d^{4} M_{0}}{d x^{2} d y^{2}}+4 x y^{3} \frac{d^{4} M_{0}}{d x d y^{3}}+y^{4} \frac{d M_{0}}{d y^{4}}\right) \\
& +\frac{\mathrm{I}}{5 !}(\text {. } \\
& +\frac{\mathrm{I}}{6 !}\left(x^{6} \frac{d^{6} M_{0}}{d x^{6}}+\ldots .+\mathrm{I} 5 x^{4} y^{2} \frac{d^{6} M_{0}}{d x^{4} d y^{2}}+\ldots .+\mathrm{I}_{5} x^{2} y^{4} \frac{d^{6} M_{0}}{d x^{2} d y^{4}}\right. \\
& \left.+\ldots .+y^{6} \frac{d^{6} M_{0}}{d y^{6}}\right)+\ldots
\end{aligned}
$$

The subscript attached to $M$ in the derivatives above indicates that the values of $x$ and $y$ corresponding to the circle $\mathrm{O}_{1}$ at the center of the section are to be inserted in the derivatives after differentiation.

To obtain $M_{1}$ the mean value of $M^{\prime}$ over the area of the section, which will be the mutual inductance of $\mathrm{O}_{2}$ and the coil $\mathrm{S}_{1}$, we must apply the operation indicated above in (2) to each term of (3). It will be noticed that every term containing an odd power of $x$ or $y$ will become zero when the limits are inserted, the derivatives $\frac{d^{2} M_{0}}{d x^{2}}$ etc., being constant. Applying this operation to the five terms in (3) which do not reduce to zero gives the following results:

$$
\begin{aligned}
& \frac{I}{2} \int_{-\frac{x}{2}}^{+\frac{x}{2}} \int_{-\frac{y}{2}}^{+\frac{y}{2}} x^{2} d x d y=\frac{x^{3} y}{3 \cdot 2^{3}}=\frac{x^{2}}{24} x y \\
& \frac{\mathrm{I}}{4 !} \iint x^{4} d x d y=\frac{2 x^{5} y}{4 ! 5 \cdot 2^{5}}=\frac{x^{4}}{1920} x y \\
& \frac{6}{4 !} \iint x^{2} y^{2} d x d y=\frac{24 x^{3} y^{3}}{4 ! 3^{2} \cdot 2^{6}}=\frac{x^{2} y^{2}}{576} x y
\end{aligned}
$$




$$
\begin{aligned}
& \frac{\mathrm{I}}{6 !} \iint x^{6} d x d y=\frac{2 x^{7} y}{6 ! 7 \cdot 2^{7}}=\frac{x^{6}}{\mathrm{I} 20.2688} x y \\
& \frac{\mathrm{I} 5}{6 !} \iint x^{4} y^{2} d x d y=\frac{4 x^{5} y^{3}}{6 ! 2^{8}}=\frac{x^{4} y^{2}}{\mathrm{I} 20.384} x y
\end{aligned}
$$

Four other terms having even powers of $x$ and $y$ give similar results, while nine terms in odd powers and those not written reduce to zero.

Substituting these values in equation (3) and writing $\frac{d^{2} M_{0}}{d \alpha^{2}}$ for $\frac{d^{2} M_{0}}{d y^{2}}$ etc., and $b_{1}$ and $c_{1}$ as the limits of integration in place of $x$ and $y$, respectively, we have:

$$
\begin{aligned}
M_{1}=M_{0} & +\frac{b_{1}{ }^{2}}{24} \frac{d^{2} M_{0}}{d x^{2}}+\frac{c_{1}^{2}}{24} \frac{d^{2} M_{0}}{d a^{2}}+\frac{b_{1}{ }^{4}}{\mathrm{I} 92 \mathrm{O}} \frac{d^{4} M_{0}}{d x^{4}}+\frac{c_{1}{ }^{4}}{\mathrm{I} 92 \mathrm{O}} \frac{d^{4} M_{0}}{d a^{4}} \\
& +\frac{b_{1}{ }^{2} c_{1}^{2}}{576} \frac{d^{4} M_{0}}{d x^{2} d a^{2}}+\frac{b_{1}{ }^{6}}{\mathrm{I} 2 \mathrm{O} .2688} \frac{d^{6} M_{0}}{d x^{6}}+\frac{c_{1}{ }^{6}}{\mathrm{I} 20.2688} \frac{d^{6} M_{0}}{d a^{6}} \\
& +\frac{b_{1}{ }^{2} c_{1}^{4}}{\mathrm{I} 20.384} \frac{d^{6} M_{0}}{d x^{2} d a^{4}}+\frac{b_{1}{ }^{4} c_{1}{ }^{2}}{\mathrm{I} 20.384} \frac{d^{6} M_{0}}{d x^{4} d a^{2}}+\ldots . .
\end{aligned}
$$

\section{MUTUAL INDUCTANCE OF TWO COILS.}

Equation (4) gives the mutual inductance of the circle $\mathrm{O}_{2}$ and the coil of section $b_{1} c_{1}$ and mean radius $\alpha$. But we wish to obtain the mutual inductance of two coils of rectangular section, and hence the above operation must be applied again.

$M_{0}$ being the mutual inductance of circles $\mathrm{O}_{1}$ and $\mathrm{O}_{2}$ at the centers of the sections $\mathrm{S}_{1}$ and $\mathrm{S}_{2}$, and $M_{1}$ being the mutual inductance of coil $S_{1}$ and circle $\mathrm{O}_{2}$, let $M_{2}$ be the mutual inductance of coil $\mathrm{S}_{1}$ and coil $\mathrm{S}_{2}$. We can now

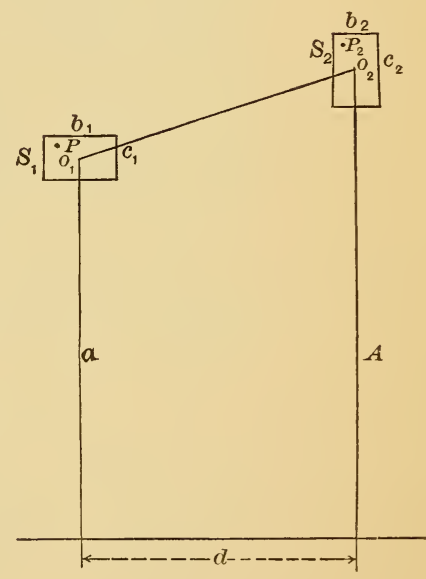

Fig. 2 obtain $M_{2}$ from $M_{1}$ in the same way that we have obtained $M_{1}$ from $M_{0}$. In this case the variable coordinates $A$ and $x$ of the circle $\mathrm{P}_{2}$ will appear in the differential coefficients, $A$ occurring where 
$a$ appears in (4), and $M_{1}$ replacing $M_{0}$, while the limits of integration are $b_{2}$ and $c_{2}$. Thus we obtain

$$
\begin{aligned}
M_{2}=M_{1} & +\frac{b_{2}{ }^{2}}{24} \frac{d^{2} M_{1}}{d x^{2}}+\frac{c_{2}{ }^{2}}{24} \frac{d^{2} M_{1}}{d A^{2}}+\frac{b_{2}{ }^{4}}{\mathrm{I} 92 \mathrm{O}} \frac{d^{4} M_{1}}{d x^{4}}+\frac{c_{2}{ }^{4}}{\mathrm{I} 92 \mathrm{O}} \frac{d^{4} M_{1}}{d A^{4}} \\
& +\frac{b_{2}{ }^{2} c_{2}{ }^{2}}{576} \frac{d^{4} M_{1}}{d x^{2} d A^{2}}+\frac{b_{2}{ }^{6}}{\mathrm{I} 2 \mathrm{O} .2688} \frac{d^{6} M_{1}}{d x^{6}}+\frac{c_{2}{ }^{6}}{\mathrm{I} 2 \mathrm{O} .2688} \frac{d^{6} M_{1}}{d A^{6}} \\
& +\frac{b_{2}{ }^{2} c_{2}{ }^{4}}{\mathrm{I} 2 \mathrm{O} \cdot 3^{8} 84} \frac{d^{6} M_{1}}{d x^{2} d A^{4}}+\frac{b_{2}{ }^{4} c_{2}{ }^{2}}{\mathrm{I} 2 \mathrm{O} .384} \frac{d^{6} M_{1}}{d x^{4} d A^{2}}+\ldots .
\end{aligned}
$$

We must now substitute the value of $M_{1}$ given by equation (4) in each term of (5), omitting derivatives higher than the sixth.

Thus:

$$
\begin{aligned}
& M_{2}=M_{0}+\frac{b_{1}{ }^{2} d^{2} M_{0}}{24} \frac{c_{1}{ }^{2} d^{2} M_{0}}{24}+\frac{b_{1}{ }^{4} d^{4} M_{0}}{1920 d a^{2}}+\frac{c_{1}^{4} d^{4} M_{0}}{1920 d a^{4}} \\
& +\frac{b_{1}{ }^{2} c_{1}{ }^{2} d^{4} M_{0}}{576 d x^{2} d a^{2}}+\frac{b_{1}{ }^{6}}{\mathrm{I} 20.2688} \frac{d^{6} M_{0}}{d x^{6}}+\frac{c_{1}{ }^{6}}{\mathrm{I} 20.2688} \frac{d^{6} M_{0}}{d a^{6}} \\
& +\frac{b_{1}{ }^{2} c_{1}^{4}}{\mathrm{I} 20.384 d x^{2} d a^{4}} \\
& +\frac{b_{1}^{4} c_{1}^{2}}{\mathrm{I} 20.384 d x^{4} d a^{2}}+\frac{d^{6} M_{0}}{24} \frac{b^{2} d^{2} M_{0}}{d x^{2}}+\frac{b_{1}{ }^{2} b_{2}{ }^{2} d^{4} M_{0}}{576 \frac{b_{2}{ }^{2} c_{1}{ }^{2} d^{4} M_{0}}{576 d x^{4} d a^{2}}} \\
& +\frac{c_{1}^{4} b_{2}{ }^{2}}{24.1920} \frac{d^{6} M_{0}}{d x^{2} d a^{4}}+\frac{b_{1}{ }^{4} b_{2}{ }^{2} d^{6} M_{0}}{24 . \mathrm{I} 920 \mathrm{O} d x^{6}}+\frac{b_{1}{ }^{2} b_{2}{ }^{2} c_{1}{ }^{2} d^{6} M_{0}}{24.576 d x^{4} d a^{2}}+\frac{c_{2}{ }^{2}}{24} \frac{d^{2} M_{0}}{d A^{2}} \\
& +\frac{c_{1}^{2} c_{2}^{2}}{576} \frac{d^{4} M_{0}}{d a^{2} d A^{2}}+\frac{b_{1}^{2} c_{2}{ }^{2}}{576} \frac{d^{4} M_{0}}{d x^{2} d A^{2}}+\frac{c_{1}^{4} c_{2}{ }^{2} d^{6} M_{0}}{24 \cdot \mathrm{I} 92 \mathrm{O} d a^{4} d A^{2}} \\
& +\frac{b_{1}^{2} c_{1}^{2} c_{2}^{2}}{24.576} \frac{d^{6} M_{0}}{d x^{2} d a^{2} d A^{2}} \\
& +\frac{b_{1}{ }^{4} c_{2}{ }^{2}}{24 . \mathrm{I} 92 \mathrm{O}} \frac{d^{6} M_{0}}{d x^{4} d A^{2}}+\frac{c_{2}{ }^{4}}{\mathrm{I} 92 \mathrm{O}} \frac{d^{4} M_{0}}{d A^{4}}+\frac{c_{1}{ }^{2} c_{2}{ }^{4}}{24 . \mathrm{I} 92 \mathrm{O}} \frac{d^{6} M_{0}}{d a^{2} d A^{4}} \\
& +\frac{b_{1}^{2} c_{2}^{4}}{24 \cdot 1920} \frac{d^{6} M_{0}}{d x^{2} d A^{4}} \\
& +\frac{b_{2}{ }^{4}}{1920} \frac{d^{4} M_{0}}{d x^{4}}+\frac{b_{2}{ }^{4} c_{1}{ }^{2}}{24 \cdot 1920} \frac{d^{6} M_{0}}{d x^{4} d a^{2}}+\frac{b_{1}{ }^{2} b_{2}{ }^{4}}{24 \cdot 1920} \frac{d^{6} M_{0}}{d x^{6}} \\
& +\frac{b_{2}^{2} c_{2}^{2}}{576} \frac{d^{4} M_{0}}{d x^{2} d A^{2}} \\
& +\frac{b_{2}{ }^{2} c_{1}{ }^{2} c_{2}{ }^{2}}{24.576} \frac{d^{6} M_{0}}{d x^{2} d \alpha^{2} d A^{2}}+\frac{b_{1}{ }^{2} b_{2}{ }^{2} c_{2}{ }^{2}}{24.576} \frac{d^{6} M_{0}}{d x^{4} d A^{2}}+\frac{c_{2}{ }^{6}}{\mathrm{I} 20.2688} \frac{d^{6} M_{0}}{d A^{6}} \\
& +\frac{b_{2}{ }^{6} d^{6} M_{0}}{\mathrm{I} 2 \mathrm{O} .2688 d x^{6}}+\frac{b_{2}{ }^{2} c_{2}{ }^{4}}{\mathrm{I} 20.384} \frac{d^{6} M_{0}}{d x^{2} d A^{4}}+\frac{b_{2}{ }^{4} c_{2}{ }^{2}}{\mathrm{I} 2 \mathrm{O} .384} \frac{d^{6} M_{0}}{d x^{4} d A^{2}}+\ldots
\end{aligned}
$$


The first Io terms of equation (6) come from substituting for $M_{1}$ in (5) its value given by (4). The last four terms are the last four terms of (5). The remaining terms come from substituting in the first five differential terms of (5) the value of $M_{1}$ in (4). Collecting like terms together equation (6) may be written

$$
\begin{aligned}
& M_{2}=M_{0}+\frac{\mathrm{I}}{24}\left\{\left(b_{1}^{2}+b_{2}^{2}\right) \frac{d^{2} M_{0}}{d x^{2}}+c_{1}{ }^{2} \frac{d^{2} M_{0}}{d a^{2}}+c_{2}{ }^{2} \frac{d^{2} M_{0}}{d A^{2}}\right\} \\
& +\frac{\mathrm{I}}{\mathrm{I} 92 \mathrm{O}}\left\{\left(b_{1}{ }^{4}+b_{2}{ }^{4}\right) \frac{d^{4} M_{0}}{d x^{4}}+c_{1}{ }_{1} \frac{d^{4} M_{0}}{d a^{4}}+c_{2}{ }^{4} \frac{d^{4} M_{0}}{d A^{4}}\right\} \\
& +\frac{\mathrm{I}}{576}\left\{b_{1}{ }^{2} b_{2}{ }^{2} \frac{d^{4} M_{0}}{d x^{4}}+c_{1}{ }^{2} c_{2}{ }^{2} \frac{d^{4} M_{0}}{d a^{2} d A^{2}}\right\} \\
& +\frac{\mathrm{I}}{576}\left(b_{1}{ }^{2}+b_{2}{ }^{2}\right)\left\{c_{1}{ }^{2} \frac{d^{4} M_{0}}{d x^{2} d a^{2}}+c_{2}{ }^{2} \frac{d^{4} M_{0}}{d x^{2} d A^{2}}\right\} \\
& +\frac{\mathrm{I}}{\mathrm{I} 20.384}\left\{\left(\frac{b_{1}{ }^{6}}{7}+b_{1}{ }^{4} b_{2}{ }^{2}+b_{1}{ }^{2} b_{2}{ }^{4}+\frac{b_{2}{ }^{6}}{7}\right) \frac{d^{6} M_{0}}{d x^{6}}\right\} \\
& +\frac{\mathrm{I}}{\mathrm{I} 2 \mathrm{O} .384}\left\{\frac{c_{1}^{6}}{7} \cdot \frac{d^{6} M_{0}}{d a^{6}}+\frac{c_{2}{ }^{6}}{7} \frac{d^{6} M_{0}}{d A^{6}}+c_{1}{ }^{2} c_{2}{ }^{4} \frac{d^{6} M_{0}}{d a^{2} d A^{4}}+c_{1}{ }^{4} c_{2}{ }^{2} \frac{d^{6} M_{0}}{d a^{4} d A^{2}}\right\} \\
& +\frac{\mathrm{I}}{\mathrm{I} 20.384}\left\{\left(b_{1}{ }^{2}+b_{2}^{2}\right) c_{1}{ }^{4} \frac{d^{6} M_{0}}{d x^{2} d a^{4}}+\left(b_{1}{ }^{2}+b_{2}^{2}\right) c_{2}{ }^{4} \frac{d^{6} M_{0}}{d x^{2} d A^{4}}\right\} \\
& +\frac{\mathrm{I}}{\mathrm{I} 2 \mathrm{O} .384}\left\{\left(b_{1}{ }^{4}+b_{2}{ }^{4}\right) c_{1}{ }^{2} \frac{d^{6} M_{0}}{d x^{4} d a^{2}}+\left(b_{1}{ }^{4}+b_{2}{ }^{4}\right) c_{2}{ }^{2} \frac{d^{6} M_{0}}{d x^{4} d A^{2}}\right\} \\
& +\frac{5}{120.576}\left\{\left(b_{1}^{2}+b_{2}^{2}\right) c_{1}^{2} c_{2}^{2} \frac{d^{6} M_{0}}{d x^{2} d a^{2} d A^{2}}\right. \\
& \left.+b_{1}{ }^{2} b_{2}{ }^{2}\left(c_{1}{ }^{2} \frac{d^{6} M_{0}}{d x^{4} d a^{2}}+c_{2}{ }^{2} \frac{d^{6} M_{0}}{d x^{4} d A^{2}}\right)\right\}
\end{aligned}
$$

Equation (7) is very much simplified by taking the two coils of equal radii, and equal section. Thus, let

$$
A=a, b_{1}=b_{2}=b, c_{1}=c_{2}=c
$$

We then have

$$
\begin{aligned}
M= & M_{0}+\frac{\mathrm{I}}{\mathrm{I} 2}\left(b^{2} \frac{d^{2} M_{0}}{d x^{2}}+c^{2} \frac{d^{2} M_{0}}{d a^{2}}\right)+\frac{\mathrm{I}}{360}\left(b^{4} \frac{d^{4} M_{0}}{d x^{4}}+c^{4} \frac{d^{4} M_{0}}{d a^{4}}\right) \\
& +\frac{\mathrm{I}}{\mathrm{I} 44}\left(b^{2} c^{2} \frac{d^{4} M_{0}}{d x^{2} d a^{2}}\right) \\
+ & \frac{\mathrm{I}}{\mathrm{I} 20.5 \mathrm{O} 4}\left(3 b^{6} \frac{d^{6} M_{0}}{d x^{6}}+3 c^{6} \frac{d^{6} M_{0}}{d a^{6}}+\mathrm{I} 4 b^{2} c^{4} \frac{d^{6} M_{0}}{d x^{2} d a^{4}}+\mathrm{I} 4 b^{4} c^{2} \frac{d^{6} M_{0}}{d x^{4} d a^{2}}\right)
\end{aligned}
$$


Equation (7) corresponds to the equation near the top of page 346 of Weinstein's article. I have omitted the factor $n_{1} n_{2}$, by which $(7)$ and (8) 1nust be multiplied if there are $n_{1}$ and $n_{2}$ turns of wire in the two coils, respectively; that is, I have so far assumed that there is only one turn of wire in each coil. . The third line of equation (7), viz,

$$
\frac{\mathrm{I}}{576}\left(b_{1}{ }^{2} b_{2}{ }^{2} \frac{d^{4} M_{0}}{d x^{4}}+c_{1}{ }^{2} c_{2}{ }^{2} \frac{d^{4} M_{0}}{d a^{2} d A^{2}}\right)
$$

is absent altogether from Weinstein's expression, and this is the source of the error in the equation which he derived from this expression for the mutual inductance of the two coils. Weinstein does not use the sixth differentials, and hence he has only the first part of equation (8). Because of the omission of the above terms the coefficient of the third term of equation (8) is in Weinstein's expression $\frac{I}{960}$ instead of $\frac{I}{360 .}$

We may write equations (4), (5), and (6), as

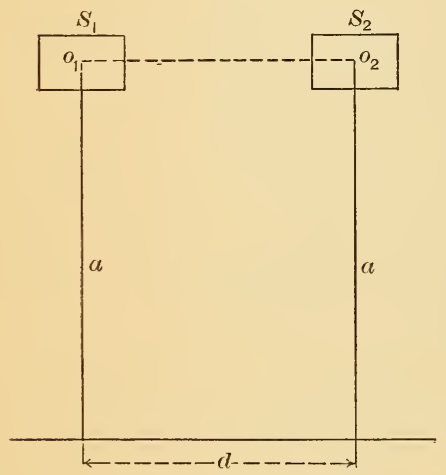

Fig. 3

$$
\begin{aligned}
M_{1} & =M_{0}+\Delta_{1} M \\
& M_{2}=M_{1}+\Delta_{2} M \\
\therefore \quad M_{2} & =M_{0}+\Delta_{1} M+\Delta_{2} \ddot{M}
\end{aligned}
$$

Supposing the two coils equal, $M_{0}$ is the mutual inductance of circle $\mathrm{O}_{1}$ and circle $\mathrm{O}_{2} ; M_{1}$ is the inductance of circle $\mathrm{O}_{1}$ on coil $\mathrm{S}_{2}$ or of $\mathrm{O}_{2}$ on $\mathrm{S}_{1} ; M_{2}$ is the inductance of $\mathrm{S}_{1}$ on $\mathrm{S}_{2}$. Therefore, $\Delta_{1} M$ is the difference between the inductance of $\mathrm{O}_{1}$ on $\mathrm{S}_{2}$ and that of $\mathrm{O}_{1}$ on $\mathrm{O}_{2}$; while $\triangle_{2} M$ is the difference between the inductance of coil $\mathrm{S}_{1}$ on $\mathrm{S}_{2}$, and $\mathrm{O}_{1}$ on $\mathrm{S}_{2}$. Obviously $\Delta_{1} M$ and $\Delta_{2} M$ are nearly equal, and when both are very small the difference can be neglected. Weinstein neglected this difference, and so took $\Delta M$ as $2 \Delta_{1} M$. He therefore merely doubled the value of $\Delta_{1} M$ given by equation (4) first line, instead of substituting it in (5) as is done above to obtain the more accurate value given in (7) and (8). This is the source of the difference between his results and those obtained in this paper. 


\section{REVISION OF WEINSTEIN'S FORMULA.}

In order to simplify the process of differentiation required in obtaining the various derivatives $\frac{d^{2} M_{0}}{d x^{2}}$. (7), not including the terms involving the sixth differentials, by means of the following relations:

$$
\begin{aligned}
\frac{d^{2} M}{d a^{2}} & =-\frac{d^{2} M}{d x^{2}}+\frac{\mathrm{I}}{a} \frac{d M}{d a} \\
\frac{d^{1} M}{d a^{4}} & =\frac{d^{4} M}{d x^{4}}-\frac{2}{a} \frac{d^{3}}{d x^{2}}\left(\frac{d M}{d a}\right)+\frac{\mathrm{I} d M}{a^{2} d x^{2}} \\
\frac{d^{4} M}{d a^{2} d x^{2}} & =-\frac{d^{4} M}{d x^{4}}+\frac{\mathrm{I}}{a} \frac{d^{2}}{d x^{2}}\left(\frac{d M}{d a}\right)
\end{aligned}
$$

Equation (9) is Laplaces's equation for cylindrical coordinates and holds for any point in space for case of symmetry about an axis. Equation (ro) is obtained by differentiating (9) successively and making some substitutions, and (II) comes from (9) by differentiating it twice with respect to $x$.

Substituting these values in the first line of equation (8) we have for the case of two coils of equal radii and equal section:

$$
\begin{aligned}
& \frac{M}{n_{1} n_{2}}=M_{0}+\left\{\frac{c^{2}}{\mathrm{I} 2 a} \frac{d M_{0}}{d a}+\frac{\mathrm{I}}{\mathrm{I} 2}\left(b^{2}-c^{2}+\frac{c^{4}}{30 a^{2}}\right) \frac{d^{2} M_{0}}{d x^{2}}\right. \\
& \left.+\frac{c^{2}}{36 a}\left(\frac{5 b^{2}-4 c^{2}}{20}\right) \frac{d^{3} M_{0}}{d x^{2} d a}+\frac{\mathrm{I}}{72}\left(\frac{b^{4}+c^{4}}{5}-\frac{b^{2} c^{2}}{2}\right) \frac{d^{4} M_{0}}{d x^{4}} \cdots\right\}(I)
\end{aligned}
$$

Equation (I2) corresponds to the following equation of Weinstein (at the middle of p. 349 of his article), $b$ and $c$ the axial breadth and radial depth of the coil corresponding to $a$ and $\rho$, and $x$ being the same as $h$.

$$
\begin{aligned}
\frac{M}{n_{1} n_{2}}=M_{0}+ & \left\{\frac{\rho^{2}}{\mathrm{I} 2 a} \frac{d M_{0}}{d a}+\frac{\mathrm{I}}{\mathrm{I} 2}\left(a^{2}-\rho^{2}+\frac{\rho^{4}}{8 \mathrm{O} a^{2}}\right) \frac{d^{2} M_{0}}{d h^{2}}\right. \\
& \left.-\frac{\rho^{2}}{48 a}\left(\frac{\rho^{2}}{\mathrm{IO}}-\frac{a^{2}}{3}\right) \frac{d^{3} M_{0}}{d h^{2} d a}+\frac{\mathrm{I}}{48}\left(\frac{\rho^{4}+a^{4}}{20}-\frac{a^{2} \rho^{2}}{3}\right) \frac{d^{4} M_{0}}{d h^{4}} \ldots\right\}
\end{aligned}
$$


(The numerator of the fraction $\frac{\rho^{4}}{8 \mathrm{O} a^{2}}$ is erroneously printed $\rho^{2}$ in Weinstein's paper.)

It will be seen that the coefficients in parenthesis of the several differential coefficients in equation (I2) differ considerably from Weinstein's in (1 $2 a)$.

Maxwell's equation for the mutual inductance of two coaxial circles is

where

$$
M=4 \pi \sqrt{A a}\left\{\left(\frac{2}{k}-k\right) F-\frac{2}{k} E\right\}
$$

$$
k=\sin \gamma=\frac{2 \sqrt{A a}}{\sqrt{(A+a)^{2}+d^{2}}}
$$

and $F$ and $E$ are complete elliptic integrals to modulus $k ; A$ and $a$ are the radii of the circles and $d$ is their distance apart. (In Weinstein's notation $\gamma$ is $\lambda, d$ is $h$, and $F$ is $K$.)

Weinstein derived the various differential coefficients of (I $2 a$ ) by differentiating ( 13 ); substituting these values in (I $2 a$ ) he obtained an equation for $M$ designated $\left(\mathrm{I}_{1}\right)$ page $350 . \quad M$ is the mutual inductance of two parallel coils of equal radii and equal section. Weinstein's formula (using his notation) is as follows:

$$
\begin{array}{r}
M=C[K-E]\left\{\epsilon+\frac{\cos ^{2} \lambda}{\mathrm{I} 2 h^{2}}\left(a_{1}-a_{2}-a_{3}+\left(2 \alpha_{2}-3 a_{3}\right) \cos ^{2} \lambda+8 a_{3} \cos ^{4} \lambda\right)\right\} \\
-C E\left\{\mathrm{I}-\frac{\sin ^{2} \lambda}{\mathrm{I} 2 h^{2}}\left(\alpha_{1}+\frac{a_{2}}{2}+2 \alpha_{3}+\left(2 \alpha_{2}+3 \alpha_{3}\right) \cos ^{2} \lambda+8 a_{3} \cos ^{4} \lambda\right)\right\}
\end{array}
$$

In the above formula $C=2 \pi d n_{1} n_{2} \sin \lambda$, where $d$ is the mean diameter of the coils $(=2 a), n_{1}$ and $n_{2}$ are the number of turns of wire in the two coils respectively, and $\lambda$ is the same as $\gamma$ above. The mean radius being $\alpha$, and the distance between the mean planes of the coils $h$,

$$
\sin ^{2} \lambda=\frac{4 a^{2}}{4 a^{2}+h^{2}}, \cos ^{2} \lambda=\frac{h^{2}}{4 a^{2}+h^{2}}
$$

$K$ and $E$ are complete elliptic integrals (identical with $F$ and $E$ of 13 ), 
$a=$ axial breadth of the cross section of the two equal coils. $\rho=$ radial depth of the cross section of the two equal coils.

The four constants $\alpha_{1}, \alpha_{2}, \alpha_{3}, \alpha_{4}$ are defined by the following expressions :

$$
\begin{gathered}
a_{1}=a^{2}-\rho^{2}+\frac{\rho^{4}}{20 d^{2}} \\
a_{2}=-\frac{\rho^{2}}{d^{2}}\left(\frac{\rho^{2}}{\mathrm{IO}}-\frac{a^{2}}{3}\right) \\
a_{3}=\frac{3}{4 h^{2}}\left(\frac{a^{4}+\rho^{4}}{2 \mathrm{O}}-\frac{a^{2} \rho^{2}}{3}\right) \\
E=\mathrm{I}+\frac{2 h^{2}}{d^{2}}+\frac{\rho^{2}}{6 d^{2}}
\end{gathered}
$$

Putting the mutual inductance of a pair of coils equal to the mutual inductance of the two circles at their center of section plus a correction due to the section we have

$$
M=M_{0}+\Delta M
$$

$M_{0}$ is then given by equation $\mathrm{I} 3$ (or any suitable formula), and $\Delta M$ will be the correction, plus or minus, depending on the size and shape of the section, the radius of the coils and their distance apart. If, then, we subtract (I3) from (I4) we shall have an expression for $\Delta M$, which is more convenient to use in calculation than (I4).

Putting

$$
\left.\begin{array}{l}
A=\frac{\cos ^{2} \lambda}{\mathrm{I} 2 h^{2}}\left(a_{1}-a_{2}-a_{3}+\left(2 a_{2}-3 a_{3}\right) \cos ^{2} \lambda+8 a_{3} \cos ^{4} \lambda\right) \\
B=\frac{\sin ^{2} \lambda}{\mathrm{I} 2 h^{2}}\left(a_{1}+\frac{a_{2}}{2}+2 a_{3}+\left(2 a_{2}+3 a_{3}\right) \cos ^{2} \lambda+8 a_{3} \cos ^{4} \lambda\right)
\end{array}\right\}
$$

we have, subtracting (I 3 ) from (I4),

$$
\Delta M=4 \pi a n_{1} n_{2} \sin \lambda\left\{(K-E)\left(A+\frac{\rho^{2}}{6 d^{2}}\right)+E B\right\}
$$

Instead of using the values of the quantities $\alpha_{1}, \alpha_{2}, \alpha_{3}$, given by (I5) we should use the values of these coefficients yielded by (I 2). Thus, in the notation of this article, putting $a_{1}, a_{2}, a_{3}$, in place of $a_{1}, a_{2}, a_{3}$, we have 


$$
\left.\begin{array}{c}
a_{1}=b^{2}-c^{2}+\frac{c^{4}}{30 a^{2}} \quad a_{2}=\frac{5 b^{2} c^{2}-4 c^{4}}{60 a^{2}} \quad a_{3}=\frac{2 b^{4}+2 c^{4}-5 b^{2} c^{2}}{20 d^{2}} \\
A=\frac{\cos ^{2} \gamma}{\mathrm{I} 2 d^{2}}\left(a_{1}-a_{2}-a_{3}+\left(2 a_{2}-3 a_{3}\right) \cos ^{2} \gamma+8 a_{3} \cos ^{4} \gamma\right) \\
B=\frac{\sin ^{2} \gamma}{\mathrm{I} 2 d^{2}}\left(a_{1}+\frac{a_{2}}{2}+2 a_{3}+\left(2 a_{2}+3 a_{3}\right) \cos ^{2} \gamma+8 a_{3} \cos ^{4} \gamma\right)
\end{array}\right\}
$$

We have now as the final expression for calculating $\Delta M$, the correction for section,

$$
\frac{\Delta M}{\pi n_{1} n_{2}}=4 \alpha \sin \gamma\left\{(F-E)\left(A+\frac{c^{2}}{24 \alpha^{2}}\right)+E B\right\}
$$

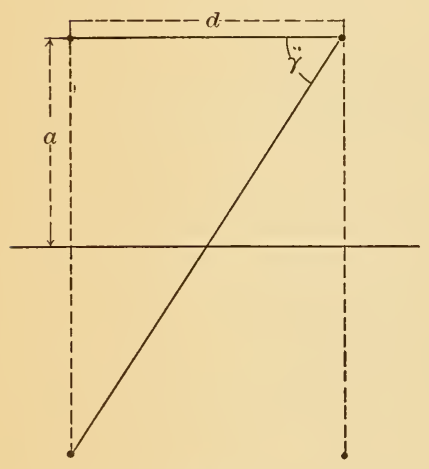

Fig. 4

In equation (20) $a$ is as before the mean radius of the two equal coils, and $\gamma$ and the other symbols have the same meaning as heretofore. Illustrations and tests of this formula will be given later in this paper.

\section{INVESTIGATION OF STEFAN'S FORMULA.}

Stefan's formula for the mutual inductance of two coaxial coils of equal radii and equal rectangular section is as follows :

$$
\left.\begin{array}{c}
M=4 \pi a n^{2}\left\{\log \frac{8 a}{d}-2+\frac{b^{2}-c^{2}}{\mathrm{I} 2 d^{2}}+\frac{2 b^{4}+2 c^{4}-5 b^{2} c^{2}}{\mathrm{I} 20 d^{4}}+\right. \\
\frac{3 b^{6}-7 b^{4} c^{2}+7 b^{2} c^{4}-3 c^{6}}{504 d^{6}}+\left(\log \frac{8 a}{d}-2\right)\left(\frac{3 b^{2}+c^{2}+\mathrm{I} 8 d^{2}}{96 a^{2}}-\frac{\mathrm{I} 5 d^{4}}{\mathrm{IO} 24 a^{4}}\right) \\
\left.+\frac{7 b^{2}+23 c^{2}+60 d^{2}}{\mathrm{I} 92 a^{2}}-\frac{29 d^{4}}{2048 a^{4}}\right\}
\end{array}\right\}
$$

The letters, $a, b, c$, and $d$ indicate as before the mean radius, the dimensions of the section and the distance apart of the mean planes of the coils.

Equation (2I) can be written as follows, omitting the factor $n^{2}$,

$$
\begin{gathered}
M=4 \pi a\left\{\log \frac{8 a}{d}\left(\mathrm{I}+\frac{3 d^{2}}{\mathrm{I} 6 a^{4}}+\frac{3 b^{2}+c^{2}}{96 a^{2}}-\frac{\mathrm{I} 5 d^{4}}{\mathrm{IO} 24 a^{4}}\right)-2-\frac{d^{2}}{\mathrm{I} 6 a^{2}}+\frac{3 \mathrm{I} d^{4}}{2048 a^{4}}\right. \\
\left.+\frac{b^{2}-c^{2}}{\mathrm{I} 2 d^{2}}+\frac{\mathrm{I} 9 c^{2}-5 b^{2}}{\mathrm{I} 92 a^{2}}+\frac{2 b^{4}+2 c^{4}-5 b^{2} c^{2}}{\mathrm{I} 2 \mathrm{O} d^{4}}+\frac{3 b^{6}-7 b^{4} c^{2}+7 b^{2} c^{4}-3 c^{6}}{504 d^{6}}\right\}
\end{gathered}
$$


The mutual inductance of two coaxial circles of equal radii is

$M_{0}=4 \pi a\left\{\log \frac{8 a}{d}\left(\mathrm{I}+\frac{3 d^{2}}{\mathrm{I} 6 a^{2}}-\frac{\mathrm{I} 5 d^{4}}{\mathrm{IO} 24 a^{4}}+..\right)-2-\frac{d^{2}}{\mathrm{I} 6 a^{2}}+\frac{3 \mathrm{I} d^{4}}{2048 a^{4}}+..\right\}(23)$

Subtracting (23) from (22) and putting as before $\Delta M$ for the difference between the mutual inductances of the two coils and of the two circles at their centers,

$$
\begin{gathered}
\Delta M=4 \pi a\left\{\frac{\frac{3 b^{2}+c^{2}}{96 a^{2}} \log \frac{8 a}{d}+\frac{\mathrm{I} 9 c^{2}-5 b^{2}}{\mathrm{I} 92 a^{2}}+\frac{b^{2}-c^{2}}{\mathrm{I} 2 d^{2}}}{504 d^{6}}\right\} \\
\left.+\frac{2 b^{4}+2 c^{4}-5 b^{2} c^{2}}{\mathrm{I} 20 d^{4}}+\frac{3 b^{6}-7 b^{4} c^{2}+7 b^{2} c^{4}-3 c^{6}}{504}\right\}(24)
\end{gathered}
$$

This expression for $\Delta M$ derived from Stefan's equation does not (as already stated) agree either with the expression (I 7 ) derived from Weinstein's equation or with the revised expression (20).

\section{DERIVATION OF NEW FORMULA.}

To derive the formula anew we must find the values of the differential coefficients of equation (8), by differentiating the series formula for the mutual inductance of two coaxial circles of unequal radii. Substituting in (8) will then give an expression for $\triangle M$. Weinstein did this as we have seen by using the expression (I3) in elliptic integrals. Stefan must have used the series formula, differentiating up to the sixth order for the principal terms. The mutual inductance of two coaxial circles of radii $a$ and $a+y$ is given by the following series: ${ }^{7}$

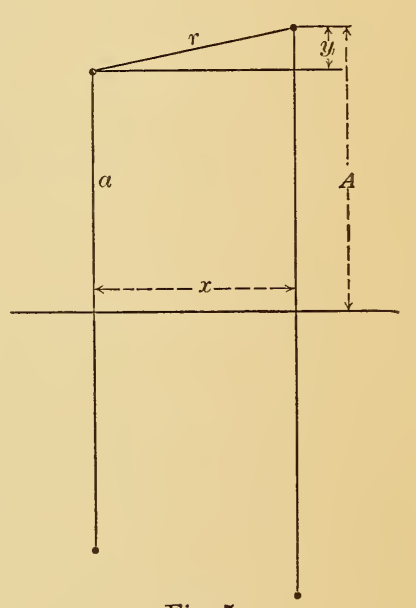

Fig. 5

$$
\begin{aligned}
& M=4 \pi a\left\{\operatorname { l o g } \frac { 8 a } { r } \left(\mathrm{I}+\frac{y}{2 a}+\frac{3 x^{2}+y^{2}}{\mathrm{I} 6 a^{2}}-\frac{3 x^{2} y+y^{3}}{32 a^{3}}-\frac{\mathrm{I} 5 x^{4}-42 x^{2} y^{2}-\mathrm{I} 7 y^{4}}{\mathrm{IO} 24 a^{4}}\right.\right. \\
& \left.+\frac{45 x^{4} y-30 x^{2} y^{3}-\mathrm{I} 9 y^{5}}{2048 a^{5}}+\ldots\right)-\left(2+\frac{y}{2 a}+\frac{x^{2}-3 y^{2}}{\mathrm{I} 6 a^{2}}-\frac{6 x^{2} y-y^{3}}{48 a^{3}}\right. \\
& \left.\left.-\frac{93 x^{4}-534 x^{2} y^{2}-\mathrm{I} 9 y^{4}}{6 \mathrm{I} 44 a^{4}}+\frac{\mathrm{I} 845 x^{4} y-3030 x^{2} y^{3}-379 y^{5}}{6 \mathrm{I} 440 a^{5}}+\ldots\right)\right\}(25)
\end{aligned}
$$


where $a$ is the radius of the smaller circle, $A=a+y$, is the radius of the larger circle, $x$ is the distance between the planes of the circles.

$$
r=\sqrt{x^{2}+y^{2}}
$$

To differentiate the entire equation at once for all the coefficients of (8) would be confusing. We may better divide it into parts and differentiate them separately. Put

$$
\begin{aligned}
& M^{\prime}=4 \pi a\left\{\left(\mathrm{I}+\frac{y}{2 a}\right) \log \frac{8 a}{r}-2-\frac{y}{2 a}\right\} \\
& M^{\prime \prime}=4 \pi a\left\{\frac{3 x^{2}+y^{2}}{\mathrm{I} 6 a^{2}} \log \frac{8 a}{r}-\frac{x^{2}-3 y^{2}}{\mathrm{I} 6 a^{2}}\right\} \\
& M^{\prime \prime \prime}=-4 \pi a\left\{\frac{3 x^{2} y+y^{3}}{32 a^{3}} \log \frac{8 a}{r}-\frac{6 x^{2} y-y^{3}}{48 a^{3}}\right\} \\
& M^{\prime \prime \prime \prime}=-4 \pi a\left\{\frac{\mathrm{I} 5 x^{4}-42 x^{2} y^{2}-\mathrm{I} 7 y^{4}}{\mathrm{IO} 24 a^{4}} \log \frac{8 a}{r}-\frac{93 x^{4}-534 x^{2} y^{2}-\mathrm{I} 9 y^{4}}{6 \mathrm{I} 44 a^{4}}\right\} \\
& M^{\prime \prime \prime \prime \prime}=4 \pi a\left\{\frac{45 x^{4} y-30 x^{2} y^{3}-\mathrm{I} 9 y^{5}}{2048 a^{5}} \cdot \log \frac{8 a}{r}-\right. \\
& \left.\frac{\mathrm{I} 845 x^{4} y-3030 x^{2} y^{3}-379 y^{5}}{61440 a^{5}}\right\}
\end{aligned}
$$

Then $M=M^{\prime}+M^{\prime \prime}+M^{\prime \prime \prime}+M^{\prime \prime \prime \prime}+M^{\prime \prime \prime \prime \prime}$

Evidently $M^{\prime}$ is the principal part of $M$ when the two circles are of nearly equal radii and near each other. As the sections of the two coils of which we are to determine the mutual inductance are supposed to be small in comparison with their radii, and the radii are to be equal, $y$ will always be relatively small. When the coils are close together $x$ will also be small. In the latter case, $M^{\prime}$ is therefore nearly the whole of $M$, and the sixth differentials required will depend almost entirely on $M^{\prime}$. On the other hand, when the coils are farther apart $M^{\prime \prime}$ is important, and we may expect an important part of the correction to depend on it, and to some extent even on $M^{\prime \prime \prime}$ and $M^{\prime \prime \prime \prime}$, but it will not be necessary to differentiate these latter expressions as far as the first.

To differentiate $M^{\prime}$ we may write it

$$
\frac{M^{\prime}}{2 \pi}=(A+a) \log \frac{8 a}{\sqrt{(A-a)^{2}+x^{2}}}-A-3 a
$$


$M^{\prime}$ must be differentiated succesively with respect to $A, a$, and $x$.

$$
\begin{gathered}
\frac{\mathrm{I} d M^{\prime}}{2 \pi d a}=\log \frac{8 a}{\sqrt{(A-a)^{2}+x^{2}}}+\frac{A^{2}-a^{2}}{(A-a)^{2}+x^{2}}+\frac{A}{a}-2 \\
\frac{\mathrm{I}}{2 \pi} \cdot \frac{d^{2} M^{\prime}}{d a^{2}}=\frac{A-a}{(A-a)^{2}+x^{2}}+\frac{\mathrm{I}}{a}-\frac{2 a}{(A-a)^{2}+x^{2}}+\frac{2(A+a)(A-a)^{2}}{\left[(A-a)^{2}+x^{2}\right]^{2}}-\frac{A}{a^{2}}
\end{gathered}
$$

when $y=A-a=0$, and $x=d$,

$$
\frac{d^{2} M_{0}^{\prime}}{d a^{2}}=4 \pi a\left[-\frac{\mathrm{r}}{d^{2}}\right]
$$

This is the value of the first differential coefficient occurring in (7) and (8) so far as it depends on $M^{\prime}$.

Differentiating $M^{\prime \prime}, M^{\prime \prime \prime}$, and $M^{\prime \prime \prime \prime}$ in a similar manner, and putting $A=a$ and $y=d$, we get for the sum of the four differential coefficients the following, omitting terms of higher power in I/ $a$ than I $a^{4}$.

$$
\begin{aligned}
& \frac{d^{2} M_{0}}{d a^{2}}=4 \pi a\left\{-\frac{\mathrm{I}}{d^{2}}+\frac{3}{\mathrm{I} 6 a^{2}}+\frac{\mathrm{I}}{8 a^{2}} \log \frac{8 a}{d}+\frac{42 d^{2}}{5 \mathrm{I} 2 a^{4}} \log \frac{8 a}{d}-\frac{\mathrm{I} 63 d^{2}}{\mathrm{IO} 24 a^{4}}\right\} \\
& \frac{d^{2} M_{0}}{d x^{2}}=4 \pi a\left\{+\frac{\mathrm{I}}{d^{2}}-\frac{\mathrm{II}}{\mathrm{I} 6 a^{2}}+\frac{3}{8 a^{2}} \log \frac{8 a}{d}-\frac{90 d^{2}}{5 \mathrm{I} 2 a^{4}} \log \frac{8 a}{d}+\frac{29 \mathrm{I} d^{2}}{\mathrm{IO} 24 a^{4}}\right\}
\end{aligned}
$$

From $(27)$ when $A=a$ and $x=d$ we have

$$
\text { I } \frac{d M_{0}^{\prime}}{d a}=4 \pi a\left\{\frac{\mathrm{I}}{2 a^{2}} \log \frac{8 \alpha}{d}-\frac{\mathrm{I}}{2 a^{2}}\right\}
$$

and if we take the same derivatives from $M^{\prime \prime}$, and $M^{\prime \prime \prime}$, and $M^{\prime \prime \prime \prime}$ and add the four values we obtain the following, omitting as before terms of higher than the fourth degree in $\mathrm{I} / a$ :

$$
\frac{\mathrm{I}}{a} \frac{d M_{0}}{d a}=4 \pi a\left\{\frac{\mathrm{I}}{2 a^{2}} \log \frac{8 a}{d}-\frac{\mathrm{I}}{2 a^{2}}+\frac{d^{2}}{8 a^{4}}-\frac{3 d^{2}}{32 a^{4}} \log \frac{8 a}{d}\right\}
$$

Equation (9) should hold in this case and give us a check on the work of differentiation, as well as a check upon the correctness of formula (25). If we have not differentiated enough terms of (25) to get all the terms of degrees contained in 29, 30, and 32 equation (9) would not be satisfied.

Equation (9) is

$$
\frac{d^{2} M}{d a^{2}}+\frac{d^{2} M}{d x^{2}}=\frac{I}{a} \frac{d M}{d a}
$$


The sum of the right hand members of (29) and (30) is

$$
4 \pi a\left\{\frac{\mathrm{I}}{2 a^{2}} \log \frac{8 a}{d}-\frac{8}{\mathrm{I} 6 a^{2}}+\frac{\mathrm{I} 28 d^{2}}{\mathrm{IO} 24 a^{4}}-\frac{48 d^{2}}{5 \mathrm{I} 2 a^{4}} \log \frac{8 a}{d}\right\}
$$

which is identically equal to (32), thus satisfying (9).

Proceeding now to the fourth differentials we find the following as the principal terms:

$$
\begin{aligned}
& \frac{d^{4} M_{0}}{d \alpha^{4}}=\frac{d^{4} M_{0}}{d A^{2}}=\frac{d^{4} M_{0}}{d x^{4}}=4 \pi a\left[+\frac{6}{d^{4}}\right] \\
& \frac{d^{4} M_{0}}{d a^{2} d x^{2}}=\frac{d^{4} M^{2}}{d A^{2} d x^{2}}=\quad 4 \pi a\left[-\frac{6}{d^{4}}\right]
\end{aligned}
$$

The expressions become complicated for these higher differentials, but we may simplify the process by transforming the equation for $M$. Thus, putting $y$ for $A-\alpha$ in equation (26), remembering that $\frac{d y}{d A}=\mathrm{I}$,

$$
\begin{gathered}
\frac{M^{\prime}}{2 \pi}=(2 a+y) \log 8 a-\left(a+\frac{y}{2}\right) \log \left(x^{2}+y^{2}\right)-(4 a+y) \\
\text { Or, } \frac{M^{\prime}}{2 \pi}=(2 a+y) \log 8 a-(4 a+y)-\left(a+\frac{y}{2}\right) \log x^{2} \\
-\left(a+\frac{y}{2}\right) \log \left(\mathrm{I}+\frac{y^{2}}{x^{2}}\right) \\
=(2 a+y) \log 8 a-(4 a+y)-(2 a+y) \log x \\
\quad-\left(a+\frac{y}{2}\right)\left[\frac{y^{2}}{x^{2}}-\frac{\mathrm{I}}{2} \frac{y^{4}}{x^{4}}+\frac{\mathrm{I}}{3} \frac{y^{6}}{x^{6}}-\ldots\right] \\
\therefore \frac{\mathrm{I}}{2 \pi} \frac{d M^{\prime}}{d A}=\log 8 a-\mathrm{I}-\log x-a\left[\frac{2 y}{x^{2}}-\frac{2 y^{3}}{x^{4}}+\frac{2 y^{5}}{x^{6}}-\ldots\right] \\
\frac{\mathrm{I}}{2}\left[\frac{3 y^{2}}{x^{2}}-\frac{5 y^{4}}{2 x^{4}}+\frac{7 y^{6}}{3 x^{6}}-\ldots\right] \\
\frac{d^{2} M^{\prime}}{d A^{2}}=-a\left[\frac{2}{x^{2}}-\frac{6 y^{2}}{x^{4}}+\frac{\mathrm{IO} y^{4}}{x^{6}}-\ldots\right]-\frac{\mathrm{I}}{2}\left[\frac{6 y}{x^{2}}-\frac{\text { Io } y^{3}}{x^{4}}+\frac{\mathrm{I} 4 y^{5}}{x^{6}}-\ldots\right] \\
\text { If } y=\mathrm{O} \text { and } x=d, \frac{d^{2} M_{0}^{\prime}}{d A^{2}}=4 \pi \alpha\left[-\frac{\mathrm{I}}{d^{2}}\right] \text { as found previously. }
\end{gathered}
$$


Proceeding with the differentiation

$$
\begin{gathered}
\frac{\mathrm{I}}{2 \pi} \frac{d^{3} M^{\prime}}{d A^{3}}=-a\left[-\frac{\mathrm{I} 2 y}{x^{4}}+\frac{40 y^{3}}{x^{6}}-\ldots\right]-\frac{\mathrm{I}}{2}\left[\frac{6}{x^{2}}-\frac{30 y^{2}}{x^{4}}+\frac{70 y^{4}}{x^{6}}-\ldots\right] \\
\frac{\mathrm{I}}{2 \pi} \frac{d^{4} M^{\prime}}{d A^{4}}=-a\left[-\frac{\mathrm{I} 2}{x^{4}}+\frac{\mathrm{I} 20 y^{2}}{x^{6}}-\ldots\right]-\frac{\mathrm{I}}{2}\left[-\frac{60 y}{x^{4}}+\frac{280 y^{3}}{x^{6}}-\ldots\right] \\
\therefore \frac{d^{4} M^{\prime}{ }_{0}}{d A^{4}}=4 \pi a\left[+\frac{6}{d^{4}}\right], \text { as found previously. }
\end{gathered}
$$$$
\frac{\mathrm{I} d^{5} M^{\prime}}{2 \pi} \frac{d A^{5}}{d}=-a\left[\frac{240 y}{x^{6}}-\ldots\right]-\frac{\mathrm{I}}{2}\left[-\frac{6 \mathrm{o}}{x^{4}}+\frac{840 y^{2}}{x^{6}}-\ldots\right]
$$$$
\frac{\mathrm{I}}{2 \pi} \frac{d^{6} M^{\prime}}{d A^{6}}=-a\left[\frac{240}{x^{6}}-\ldots .\right]-\frac{\mathrm{I}}{2}\left[\frac{\mathrm{I} 680 y}{x^{6}}-\ldots\right]
$$

All terms in the series that have been omitted have $y$ as a factor, and hence when $y=0$ they all vanish. Hence there is only one term in the entire series that does not vanish for $\frac{d^{6} M_{0}}{d A^{6}}$. Thus we have

$$
\frac{d^{6} M_{0}}{d A^{6}}=4 \pi a\left[-\frac{\mathrm{I} 2 \mathrm{O}}{d^{6}}\right]
$$

The same method is successful in obtaining the differential coefficients with respect to $a$ and $x$. The following are the values:

$$
\begin{array}{ll}
\frac{d^{6} M_{0}^{\prime}}{d a^{6}}=\frac{d^{6} M_{0}^{\prime}}{d A^{6}}=\frac{d^{6} M_{0}^{\prime}}{d x^{4} d a^{2}}=4 \pi a\left[-\frac{\text { I } 20}{d^{6}}\right] \\
\frac{d^{6} M_{0}^{\prime}}{d x^{6}}=\frac{d^{6} M_{0}^{\prime}}{d x^{2} d a^{4}}=\quad 4 \pi a\left[+\frac{\text { I } 20}{d^{6}}\right]
\end{array}
$$

We may safely neglect the fourth and sixth differentials of $M^{\prime \prime}$ and $M^{\prime \prime \prime}$, which latter are very small where the field is changing rapidly, that is, near the primary coil.

Substituting now the values of the coefficients as given by (29), (30), (35), and (37) in equation (8), in which the coils are assumed 
to have the same radii and section, we obtain the following expression for $\Delta M$ :

$$
\begin{gathered}
\Delta M=4 \pi a\left\{\frac{3 b^{2}+c^{2}}{96 a^{2}} \log \frac{8 a}{d}-\frac{\mathrm{II} b^{2}-3 c^{2}}{\mathrm{I} 92 a^{2}}+\frac{b^{2}-c^{2}}{\mathrm{I} 2 d^{2}}+\frac{2 b^{4}+2 c^{4}-5 b^{2} c^{2}}{\mathrm{I} 2 \mathrm{O} d^{4}}\right. \\
+\frac{3 b^{6}-3 c^{6}+\mathrm{I} 4 b^{2} c^{4}-\mathrm{I} 4 b^{4} c^{2}}{504 d^{6}}+\frac{7 c^{2} d^{2}}{\mathrm{IO} 24 a^{4}}\left(\log \frac{8 a}{d}-\frac{\mathrm{I} 63}{84}\right) \\
\left.-\frac{\mathrm{I} 5 b^{2} d^{2}}{\mathrm{IO} 24 a^{4}}\left(\log \frac{8 a}{d}-\frac{97}{60}\right)\right\}
\end{gathered}
$$

The second and fifth terms of the equation ( $\left.3^{8}\right)$ differ from the corresponding terms of (24) which was derived from Stefan's formula, the principal difference being in the second term, and there are two additional terms in (38). For a square section the second term is negative in (38) and positive and larger in (24). It is the error in this term chiefly which makes Stefan's formula give too large values. This is especially noticeable when the coils are far apart, when the other corrections are small. When the coils are near together the other corrections are so much larger that this error is obscured, and the formula appears more nearly correct.

Instead of substituting in (8) we might have substituted in (12) the transformed equation used by Weinstein, adding the second line of (8) to (I2) to give the terms depending on the sixth differentials. This requires the value of one other differential coefficient, viz:

$$
\text { I } \frac{d^{3} M_{0}}{d x^{2} d a}=4 \pi a\left\{\frac{\mathrm{I}}{2 a^{2} d^{2}}-\frac{3}{\mathrm{I} 6 a^{4}} \log \frac{8 a}{d}\right\}
$$

Carrying out these substitutions we obtain the same expression (38) with some additional very small terms, which are negligible, except for coils of very large section, or coils very near together, in which case one term is appreciable. This term is-

$$
\Delta_{2} M=4 \pi a\left\{\frac{6 b^{4}+6 c^{4}+5 b^{2} c^{2}}{5760 a^{2} d^{2}}\right\}
$$

and may be used if needed by adding it to (38). It is included in (4I) below. For a square section, where $b=c$, the third and fifth terms of (38) disappear and the formula becomes; adding (39): 


$$
\Delta M=4 \pi\left\{\left\{\frac{b^{2}}{24 a^{2}}\left(\log \frac{8 a}{d}-\mathrm{I}\right)-\frac{b^{2} d^{2}}{\mathrm{I} 28 a^{2}}\left(\log \frac{8 a}{d}-\frac{4}{3}\right)-\frac{b^{4}}{\mathrm{I} 20 d^{4}}+\frac{\mathrm{I} 7 b^{4}}{5760 a^{2} d^{2}}\right\}\right.
$$

$$
\text { Or, } \Delta M=\frac{\pi b^{2}}{6 a}\left\{\log \frac{8 a}{d}-\mathrm{I}-\frac{3 d^{2}}{\mathrm{I} 6 a^{2}}\left(\log \frac{8 a}{d}-\frac{4}{3}\right)-\frac{a^{2} b^{2}}{5 d^{4}}+\frac{17 b^{2}}{240 d^{2}}\right\}
$$

The following approximate formula for coils of square section is sufficiently exact for most purposes, although varying more from the exact expression as $d$ is greater:

$$
\Delta M=\frac{\pi b^{2}}{6 a}\left\{\log \frac{8 a}{d}--\mathrm{I}-\frac{a^{2} b^{2}}{5 d^{4}}\right\}
$$

When the two coils have equal radii but unequal sections substitution must be made in (7) instead of (8). Neglecting sixth differentials, which are inappreciable except for coils very near, we obtain for coils not very far apart the following expression:

$$
\begin{aligned}
& \Delta M=4 \pi a\left\{\frac{3\left(b_{1}{ }^{2}+b_{2}{ }^{2}\right)+c_{1}{ }^{2}+c_{2}{ }^{2}}{\mathrm{I}^{2} a^{2}} \log \frac{8 a}{d}-\frac{\operatorname{II}\left(b_{1}{ }^{2}+b_{2}{ }^{2}\right)-3\left(c_{1}{ }^{2}+c_{2}{ }^{2}\right)}{384 a^{2}}\right. \\
& +\frac{\left(b_{1}{ }^{2}+b_{2}{ }^{2}\right)-\left(c_{1}{ }^{2}+c_{2}{ }^{2}\right)}{24 d^{2}} \\
& \left.+\frac{\left(3 b_{1}^{4}+\operatorname{IO}_{1}^{2} b_{2}^{2}+3 b_{2}^{4}\right)+\left(3 c_{1}^{4}+\operatorname{IO}_{1}{ }^{2} c_{2}{ }^{2}+3 c_{2}^{4}\right)-\mathrm{IO}\left(b_{1}{ }^{2}+b_{2}^{2}\right)\left(c_{1}{ }^{2}+c_{2}{ }^{2}\right)}{960 d^{4}}\right\}
\end{aligned}
$$

In every case the value of $\Delta M$ is to be multiplied by $n_{1} n_{2}$ where $n_{1}$ and $n_{2}$ are the number of turns of wire on the two coils.

\section{COILS OF A SINGLE LAYER. CURRENT SHEETS.}

By putting $c=0$ in formula $\left(3^{8}\right)$ we get an expression for $\Delta M$ for two coaxial current sheets, which would be realized substantially by single layer coils, so far as mutual inductance is concerned. If we consider their radii as fixed, and therefore $a$ constant, equations (4) and (5) would be simplified by the disappearance of all terms in$16360-07-3$ 
volving differential coefficients with respect to $a$. Equation (7) would then become:

$$
\begin{aligned}
M=M_{0} & +\frac{\mathrm{I}}{24}\left(b_{1}{ }^{2}+b_{2}{ }^{2}\right) \cdot \frac{d^{2} M_{0}}{d x^{2}} \\
& +\left(\frac{b_{1}{ }^{4}+b_{2}{ }^{4}}{\mathrm{I} 92 \mathrm{O}}+\frac{b_{1}{ }^{2} b_{2}{ }^{2}}{576}\right) \frac{d^{4} M_{0}}{d x^{4}} \\
& +\left(\frac{b_{1}{ }^{6}+b_{2}{ }^{6}}{\mathrm{I} 2 \mathrm{O} 2688}+\frac{b_{1}{ }^{2} b_{2}{ }^{4}+b_{1}{ }^{4} b_{2}{ }^{2}}{24 . \mathrm{I} 92 \mathrm{O}}\right) \frac{d^{6} M_{0}}{d x^{6}} \\
& +\left(\frac{b_{1}{ }^{8}+b_{2}{ }^{8}}{\mathrm{I} 20.256 .3024}+\frac{b_{1}{ }^{6} b_{2}{ }^{2}+b_{1}{ }^{2} b_{2}{ }^{6}}{24 . \mathrm{I} 2 \mathrm{O} 268268}+\frac{b_{1}{ }^{4} b_{2}{ }^{4}}{(\mathrm{I} 92 \mathrm{O})^{2}}\right) \frac{d^{8} M_{0}}{d x^{8}}+\ldots
\end{aligned}
$$

When the coils have equal lengths, i. e., $b_{1}=b_{2}=b$,

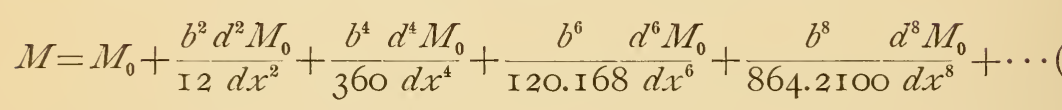

Taking Coffin's extension of Maxwell's equation for the mutual inductance of two coaxial circles of equal radius we can easily obtain the differential coefficients to the eighth order, to substitute in (45). The expression for $M$, for the two equal circles is as follows:

$$
\begin{aligned}
M=4 \pi a\left\{\log \frac{8 a}{d}\right. & \left(\mathrm{I}+\frac{3 x^{2}}{\mathrm{I} 6 a^{2}}-\frac{\mathrm{I} 5 x^{4}}{8 . \mathrm{I} 28 a^{4}}+\frac{35 x^{6}}{\mathrm{I} 28^{2} a^{6}}-\frac{\mathrm{I} 575 x^{8}}{2 \cdot \mathrm{I} 28^{3} a^{8}}+\ldots\right) \\
& -\left(2+\frac{x^{2}}{\mathrm{I} 6 a^{2}}-\frac{3 \mathrm{I} x^{4}}{\mathrm{I} 6 . \mathrm{I} 28 a^{4}}+\frac{247 x^{6}}{6 . \mathrm{I} 28^{2} a^{6}}-\frac{7,795 x^{8}}{8 \cdot \mathrm{I} 28^{3} a^{8}}+\cdots\right)(46)
\end{aligned}
$$

Because $y$ is zero, the equation is simpler to the eighth degree than equation (25) to the fifth degree, and the differentiation is greatly simplified by making $a$ constant.

The values of the differential coefficients are as follows:

$$
\begin{aligned}
& \frac{d^{2} M_{0}}{d x^{2}}=4 \pi a\left\{\log \frac{8 a}{d} \cdot\left(\frac{3}{8 a^{2}}-\frac{45 d^{2}}{256 a^{4}}+\frac{\mathrm{IO} 50 d^{4}}{\mathrm{I} 28^{2} a^{6}}-\frac{44 \mathrm{IOO} d^{6}}{\mathrm{I} 28^{3} a^{8}} \ldots\right)\right. \\
& \frac{d^{4} M_{0}}{d x^{4}}=4 \pi a\left\{\frac{3}{8 a^{2} d^{2}}+\frac{6}{d^{4}}-\frac{45}{\mathrm{I} 28 a^{4}}\left(\log \frac{8 a}{d}-\frac{\mathrm{I} 87}{60}\right)\right\} \\
& \frac{d^{6} M_{0}}{d x^{6}}=4 \pi a\left\{\frac{\mathrm{I} 20}{d^{6}}\right\}
\end{aligned}
$$


$\frac{d^{8} M_{0}}{d x^{6}}=4 \pi a\left\{\frac{5040}{d^{8}}\right\}$

Substituting these values of the differential coefficients in (45) we get for $\Delta M$ the following expression:

$$
\begin{aligned}
\Delta M= & 4 \pi a\left[\frac{b^{2}}{\mathrm{I} 2 d^{2}}+\frac{b^{2}}{32 a^{2}}\left(\log \frac{8 a}{d}-\frac{\mathrm{II}}{6}\right)-\frac{\mathrm{I} 5 b^{2} d^{2}}{\mathrm{IO} 24 a^{4}}\left(\log \frac{8 a}{d}-\frac{97}{60}\right)\right. \\
& +\frac{\mathrm{I} 75 b^{2} d^{4}}{2(\mathrm{I} 28)^{2} a^{6}}\left(\log \frac{8 a}{d}-\frac{54}{35}\right)-\frac{3675 b^{2} d^{6}}{(\mathrm{I} 28)^{3} a^{8}}\left(\log \frac{8 a}{d}-\frac{3793}{2520}\right)+\frac{b^{4}}{960 a^{2} d^{2}} \\
& \left.+\frac{b^{4}}{60 d^{4}}-\frac{b^{4}}{\mathrm{IO} 24 a^{4}}\left(\log \frac{8 a}{d}-\frac{\mathrm{I} 87}{60}\right)+\frac{b^{6}}{\mathrm{I} 68 d^{6}}+\frac{b^{8}}{360 d^{8}}\right\}
\end{aligned}
$$

This formula will give the mutual inductance (adding $\Delta M$ to $M_{0}$ ) with very great precision for two coaxial single layer coils of equal radii, provided the coils are not in contact or $d$ is not too great. If $d$ is equal to or greater than $a$ we can obtain a more accurate value by using formula (20) making $c=0$. In this case

$$
\begin{gathered}
A=\frac{b^{2} \cos ^{2} \gamma}{\mathrm{I} 2 d^{2}}\left(\mathrm{I}-\frac{b^{2}}{\mathrm{IO} d^{2}}-\frac{3 b^{2}}{\mathrm{IO} d^{2}} \cos ^{2} \gamma+\frac{4 b^{2}}{5 d^{2}} \cos ^{4} \gamma\right) \\
B=\frac{b^{2} \sin ^{2} \gamma}{\mathrm{I} 2 d^{2}}\left(\mathrm{I}+\frac{b^{2}}{5 d^{2}}+\frac{3 b^{2}}{\mathrm{IO} d^{2}} \cos ^{2} \gamma+\frac{4 b^{2}}{5 d^{2}} \cos ^{4} \gamma\right) \\
\Delta M=4 \pi a \sin \gamma\{(F-E) A+E B .\}
\end{gathered}
$$

If the coils are considerably distant from one another this expression is very exact. If quite near we may improve the accuracy by adding to the value of $\Delta M$ obtained by $(52)$ the last two terms of (5I) which represent the part of $\Delta M$ depending on sixth and eighth differentials. Examples of the use of these formulæ on single layer coils will be found in another paper in this bulletin. ${ }^{8}$

We must now test the formulæ for $\Delta M$ by numerical applications to see how closely it approximates to the true value of the correction $\Delta M$. 


\section{TESTS OF FORMULE 38 AND 41.}

Let there be two coaxial coils of mean radii $25 \mathrm{~cm}$, and square section $2 \times 2 \mathrm{~cm}$, and distant $4 \mathrm{~cm}$ between their mean planes. Thus

$$
\begin{aligned}
& a=25 \\
& b=2 \\
& c=2 \\
& d=4
\end{aligned}
$$$$
\log _{e} \frac{8 a}{d}=\log _{e} 5 \mathrm{O}=3.9 \mathrm{I} 20
$$

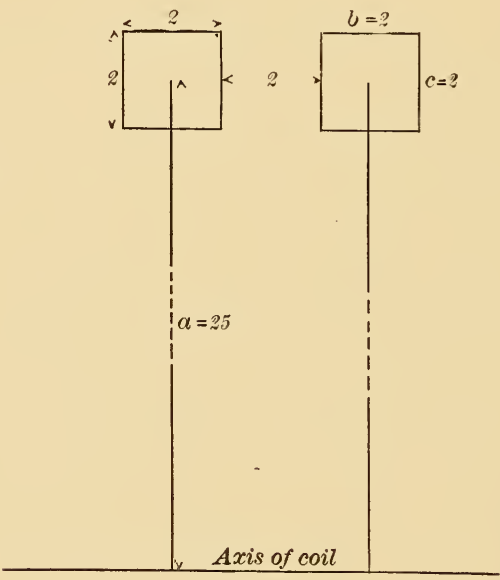

Fig. 6

Substituting these values in (4I) we have

$$
\begin{aligned}
& \log \frac{8 a}{d}-\mathrm{I} \quad=2.9120 \\
& \frac{\mathrm{I} 7 b^{2}}{240 d^{2}} \quad=\quad .0177=2.9297 \\
& -\frac{a^{2} b^{2}}{5 d^{4}} \quad=-\mathrm{I} .953 \mathrm{I} \\
& -\frac{3 d^{2}}{\mathrm{I} 6 a^{2}}\left(\log \frac{8 a}{d}-\frac{4}{3}\right)=\frac{.0 \mathrm{I} 24}{-}=\frac{-\mathrm{r} .9655}{+0.9642} \\
& \frac{b^{2}}{6 a}=\frac{4}{\mathrm{I} 5 \mathrm{O}} ; 0.9642 \times \frac{4}{\mathrm{I}_{50}}=.0257 \mathrm{I} \\
& \therefore \quad \frac{\Delta M}{\pi}=+0.0257 \mathrm{I}
\end{aligned}
$$

The value of $\frac{M_{0}}{\pi}$ as given by (13) or (23) is I 92.9 I 74 , assuming only 
one turn in each coil. The correction $\Delta M$ is therefore about one part in 7,500 . If there were 400 turns of wire in each coil the mutual inductance would be

$$
\begin{aligned}
M & =\overline{400^{2}} \pi(\text { I } 92.9 \text { I } 74+.0257) \\
& =96,983,800 \mathrm{cln} \\
& =96.983^{8} \text { millihenrys. }
\end{aligned}
$$

If the coils be moved apart farther the correction $\Delta M$ increases, and at $d=6 \mathrm{~cm}, \frac{\Delta M}{\pi}=.056 \mathrm{r} 3$ more than double its value at $4 \mathrm{~cm}$. This is contrary to what one would at first expect, until one remem-

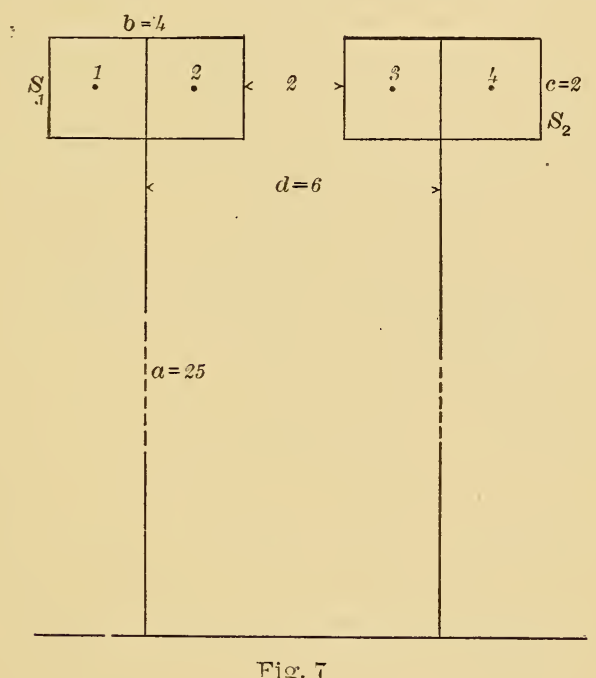

bers that for coils close together the correction is negative. At $d=3 \mathrm{~cm}$ it is still negative, while at some point between 3 and 4 it is zero. At $d=8 \mathrm{~cm}$ the correction $\frac{\Delta M}{\pi}$ is .05373 by formula (4I).

These corrections are all relatively small, and have been computed with great accuracy, giving the mutual inductances $M$ at these various distances with high precision.

Let there be two coils $S_{1}$ and $S_{2}$ each of rectangular section $2 \times 4$ $\mathrm{cm}$, and their mean planes distant $6 \mathrm{~cm}$. Each coil may be regarded as inade up of two coils of square section $2 \times 2$. The mutual induc- 
tance of the two coils $S_{1}$ and $S_{2}$ upon one another is made up of the sum of the mutual inductances of coils $I$ and 2 upon coils 3 and 4 , coils I and 2 being the two halves of $S_{1}$ and 3 and 4 the two halves of $\mathrm{S}_{2}$. Thus,

$$
M=M_{13}+M_{14}+M_{23}+M_{24}
$$

$M_{13}$ is of course equal to $M_{24}$.

We have given above the values of $\frac{\Delta M}{\pi}$ for each of these separate cases and we can now obtain the mutual inductance of $S_{1}$ on $S_{2}$. Thus

$$
\begin{aligned}
& \frac{M_{\mathrm{o}}}{\pi} \quad \frac{\Delta M}{\pi} \\
& \begin{array}{lll}
M_{13} & \text { I } 54.07 \mathrm{I} 8 & .056 \mathrm{I} 3
\end{array} \\
& \begin{array}{lll}
M_{14} & \text { I } 27.3947 & .05373
\end{array} \\
& \begin{array}{lll}
M_{23} & \text { I92.9I } 74 \quad .0257 \mathrm{I}
\end{array} \\
& M_{24}\left(M_{0} \div \pi\right) \quad \frac{\text { I } 54.07 \mathrm{I} 8}{628.4557} \quad \frac{.056 \mathrm{I} 3}{\text {.I9I70 }} \\
& \Sigma(\Delta M \div \pi) \quad \text {.I9I7 } \\
& \frac{M}{\pi}=\quad 628.6474
\end{aligned}
$$

We can now determine the value of $M$ by another process. Regarding the coils $S_{1}$ and $S_{2}$ as made up of two parts of one turn each, calculate $M_{0}$ by (I3) or (23) and $\Delta M$ by (38). The distance apart of the mean planes being $6 \mathrm{~cm}$, we see that $M_{0}$ is four times $M_{13}$, that is,

$$
\text { For } \mathrm{S}_{1} \text { on } \mathrm{S}_{2}, \frac{M_{0}}{\pi}=6 \mathrm{r} 6.2872
$$

To calculate $\Delta M$, substitute in (38) the following values:

$$
\begin{aligned}
& a=25 \\
& b=4 \\
& c=2 \\
& d=6
\end{aligned} \quad \log _{e} \frac{8 a}{d}=\log _{e} \frac{200}{6}=3.5065
$$




$$
\begin{aligned}
& \frac{3 b^{2}+c^{2}}{96 a^{2}} \log \frac{8 a}{d} \quad=.003038 \\
& \frac{b^{2}-c^{2}}{\mathrm{I} 2 d^{2}} \\
& =.027778 \\
& \frac{2 b^{4}+2 c^{4}-5 b^{2} c^{2}}{\mathrm{I} 2 \mathrm{O} d^{4}} \\
& =.001440 \\
& \frac{3 b^{6}-3 c^{6}+14 b^{2} c^{4}-14 b^{4} c^{2}}{504 d^{6}}=.000057 \\
& \frac{6 b^{4}+6 c^{4}+5 b^{2} c^{2}}{5760 a^{2} d^{2}} \quad=\text {.0000I } 5 \\
& \frac{7 c^{2} d^{2}}{\mathrm{IO}_{2} 4 a^{4}}\left(\log \frac{8 a}{d}-\frac{\mathrm{I} 63}{84}\right)=.000004=.032332 \\
& -\frac{15 b^{2}-3 c^{2}}{192 a^{2}} \quad=-.001367 \\
& \frac{-\mathrm{I} 5 b^{2} d^{2}}{\mathrm{I} 024 a^{4}}\left(\log \frac{8 a}{d}-\frac{97}{60}\right)=\underline{.000040}=\frac{-.001407}{.030925} \\
& 4 a n^{2}=400 \quad \therefore \frac{\Delta M}{\pi}=12.370 \\
& \frac{M_{0}}{\pi}=\underline{616.287} \\
& \therefore \frac{M}{\pi}=628.657
\end{aligned}
$$

By process of summation above $M \pi=628.647$

$$
\text { Difference }=\text {.oIO }
$$

which is about one part in 60,000. The correction by the process of summation is so small that we may be sure it is more exact than the second method, in which the correction is more than 2 per cent. To give absolute agreement between the results of the two methods the second correction should be I2.360 instead of I $2.37 \mathrm{O}$, a difference of less than one-tenth of one per cent of the correction. This is a very good test of the formula ( $\left.3^{8}\right)$ for this distance between the coils, and the accuracy is very satisfactory. 
TEST OF FORMULA 20.

Let formula (2O) be applied to the same coil to which $4 \mathrm{I}$ has been applied, where

$$
\begin{array}{lr}
a=25 & \sin ^{2} \gamma=\frac{2500}{25 \mathrm{I} 6} \\
b=2 & \\
c=2 & \cos ^{2} \gamma=\frac{\mathrm{I} 6}{25 \mathrm{I} 6} \\
d=4 &
\end{array}
$$

Substituting the above values of the constants in (I8) we find the following values of $a_{1}, a_{2}, a_{3}$ :

$$
\begin{aligned}
a_{1} & =.000853 \\
a_{2} & =.000427 \\
a_{3} & =-.050000 \\
\frac{c^{2}}{24 a^{2}} & =.0002667
\end{aligned}
$$

Substituting in (r9) we obtain

$$
\begin{aligned}
A+\frac{c^{2}}{24 a^{2}} & =.0002684 \\
B & =-.0005170
\end{aligned}
$$

The complete elliptic integrals to modulus $\sin \gamma$ have the following values for this particular case:

$$
\begin{gathered}
F \quad=3.91986 \\
E \quad=1.01088 \\
F-E \quad=2.90898 \\
\therefore(F-E)\left(A+\frac{c^{2}}{24 \alpha^{2}}\right)=.00078077 \\
E B=-.00052262 \\
\text { Sum }=.00025815 \\
4 a \sin \gamma=99.6815 \\
\therefore \frac{\Delta M}{\pi}=.02573
\end{gathered}
$$

Formula (4I) gave .0257I, which is almost in absolute agreement, the last figure being dropped in the final calculations. The uncertainty in this value is probably not greater than one in a million of the whole inductance of these two coils. 
Taking now the larger coils $\mathrm{S}_{1}$ and $\mathrm{S}_{1}$, Fig. (7), where $b=4$, and substituting in equation (20) we get the larger correction $\frac{\Delta M \text {. }}{\pi}$ Following the process indicated above we find for $\mathrm{S}_{1}$ and $\mathrm{S}_{2}$,

$$
\begin{aligned}
\frac{\Delta M}{\pi} & =\mathrm{I} 2.349 \\
\text { As before, } \frac{M_{0}}{\pi} & =6 \mathrm{r} 6.287 \\
\therefore M & =628.636
\end{aligned}
$$

Value by summation, 628.647

$$
\text { Difference }=\text {.OII }=\mathrm{I} \text { part in } 57, \mathrm{OOO} \text {. }
$$

This is nearly as good agreement as by formulae (38) and (4I).

There are decided advantages in calculating the quantity $\Delta M$ separately and adding it to $M_{0}$ to obtain $M$, the mutual inductance of two coils. For $M_{0}$ can be calculated by more than one process, as a check on the numerical work as well as a check on the formulæ, and similarly $\Delta M$ can be calculated by more than one formula. When they are put together in one formula one can not be sure to what any difference found between the results of two different formulæ is due. There is no trouble in computing $M_{0}$ to any desired degree of accuracy. Practically the whole problem is to find $\Delta M$. Hence it is safer as well as more convenient when practicable to have separate formulæ for $\Delta M$.

The above tests of these formulæ show that for coils of this size and distance apart very accurate values of the mutual inductance can be obtained, as accurate as will be required in the most exact experimental work. Of course, if the cross sections are greater the accuracy will be less, but in work of precision large cross sections should not be used; the depth would probably seldom be greater than $2 \mathrm{~cm}$. Formulæ (38) and (4r) give values for $\Delta M$ a little less exact as the distance apart of the coils increases, since the terms neglected in the series (25) becomes appreciable when $d$ is large. For such cases formula (2O) is more exact. Further tests and discussion of these formulæ are given in another paper $^{9}$ in this Bulletin. 


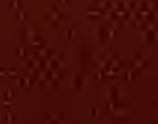

$\lim ^{2} \rightarrow 5=$

a.

2005

s.

38

scis

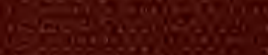

resions

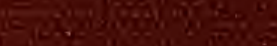

206

3.

ST

Wha

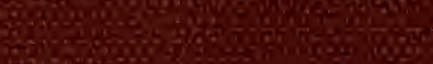

Sor. 